\title{
Occurrence statistics of cold, streaming ions in the near-Earth magnetotail: Survey of Polar-TIDE observations
}

\author{
M. W. Liemohn, ${ }^{1}$ T. E. Moore, ${ }^{2}$ P. D. Craven, ${ }^{3}$ W. Maddox, ${ }^{3}$ A. F. Nagy, ${ }^{2}$ \\ and J. U. Kozyra ${ }^{2}$ \\ Received 24 September 2004; revised 12 April 2005; accepted 18 April 2005; published 9 July 2005.
}

[1] Results are presented from a survey of cold ion observations in the near-Earth magnetotail using data from the Polar Thermal Ion Dynamics Experiment (TIDE). During the interval from July to December of 2001 , Polar had its apogee $\left(\sim 9.5 R_{E}\right)$ near the equatorial plane in the tail region of the magnetosphere. It is shown that a lobal wind is ubiquitous in the inner tail, with low-energy $(<300 \mathrm{eV})$ ions streaming from the ionosphere downtail. These lobal winds often pass through the plasma sheet, forming bidirectional streams, in addition to the unidirectional beams seen at higher magnetic latitudes. The observance of bidirectional streams is inversely, although weakly, correlated with geomagnetic activity. Bidirectional streams are interpreted as indicating the minimum size of the closed flux tube region. The reduced frequency of bidirectional streams with activity level times is consistent with the thinning of the plasma sheet during these times. It is inferred from the universality of these observations during Polar's passage through the inner tail region that the ionosphere is a continuous supplier of plasma to the near-Earth magnetosphere. The high occurrence rate of these streams means that during geomagnetic disturbances, it is not necessary to wait for outflow and magnetospheric circulation in order to supply the inner magnetosphere with ionospheric ions; these cold streams are an immediately available supply of ionospheric-origin particles.

Citation: Liemohn, M. W., T. E. Moore, P. D. Craven, W. Maddox, A. F. Nagy, and J. U. Kozyra (2005), Occurrence statistics of cold, streaming ions in the near-Earth magnetotail: Survey of Polar-TIDE observations, J. Geophys. Res., 110, A07211, doi:10.1029/ 2004JA010801.

\section{Introduction}

[2] It is known that the ionosphere supplies some amount of material to the magnetosphere [e.g., Dessler and Michel, 1966; Shelley et al., 1972, 1982; Horwitz, 1982; Chappell et al., 1987; Moore and Delcourt, 1995]. Ionospheric ions, such as $\mathrm{He}^{+}$and $\mathrm{O}^{+}$, have been observed streaming out of the high-latitude ionosphere into the polar cap region [e.g., Moore et al., 1997; Su et al., 1998; Elliott et al., 2001]. From there, they are able to flow through the tail lobes [e.g., Sharp et al., 1981; Candidi et al., 1982; Seki et al., 1998, 2000, 2003], sometimes seen hundreds of $R_{E}$ downtail [Mukai et al., 1994; Hirahara et al., 1996; Seki et al., 1996]. Observations of ionospheric ions have also been made in the plasma sheet [e.g., Peterson et al., 1981; Young et al., 1982; Lennartsson and Shelley, 1986; Orsini et al., 1990], with significant enhancements seen during storms [e.g., Lennartsson et al., 1981] and substorms [Daglis and

\footnotetext{
${ }^{1}$ Space Physics Research Laboratory, University of Michigan, Ann Arbor, Michigan, USA.

${ }^{2}$ Laboratory for Extraterrestrial Physics, NASA Goddard Space Flight Center, Greenbelt, Maryland, USA.

${ }^{3}$ National Space Science and Technology Center, NASA Marshall Space Flight Center, Huntsville, Alabama, USA.
}

Copyright 2005 by the American Geophysical Union. 0148-0227/05/2004JA010801
Axford, 1996; Nosé et al., 2001, 2003]. Energetic $\mathrm{O}^{+}$is also a big contributor to the ring current, particularly during large magnetic storms [e.g., Hamilton et al., 1988; Daglis et al., 1993; Collin et al., 1993; Pulkkinen et al., 2001; Fu et al., 2002]. Occasionally, low-energy field-aligned flows of $\mathrm{O}^{+}$(sometimes termed "zipper" events) are observed deep within the inner magnetosphere during storms [Kaye et al., 1981; Fennell et al., 1981; Cladis and Collin, 1997; Sheldon and Spence, 1997]. Oxygen ions in the ring current are thought to be primary contributors to stable auroral red arc formation [Kozyra et al., 1987, 1997]. It has been speculated that the superdense plasma sheet intervals, as seen at geosynchronous altitude, might be of ionospheric origin [Borovsky et al., 1997], although some debate about this exists [Lennartsson et al., 1981; Thomsen et al., 2003; Stubbs et al., 2004]. It has even been postulated that the ionosphere is a fully adequate source of plasma for the magnetosphere [Chappell et al., 1987, 2000; M. Huddleston et al., Understanding the Earth's ionosphere as a fully sufficient source for magnetospheric plasma, submitted to Journal of Geophysical Research, 2005, hereinafter referred to as Huddleston et al., submitted manuscript, 2005]. At the very least, it is clear that ionospheric constituents, particularly $\mathrm{O}^{+}$, can significantly contribute to the plasma sheet density and to the energy of the ring current.

[3] The existence of ionospheric plasma in the near-earth magnetotail lobes, however, has not been well documented. 
The high-latitude regions of the lobes above the polar caps have been surveyed [e.g., Moore et al., 1997; Su et al., 1998; Elliott et al., 2001], but the low- to midlatitude range of the near-Earth lobes across the nightside magnetosphere has not. This is a critical region because it is magnetically connected to the near-Earth plasma sheet and can thus rapidly supply plasma to the inner magnetosphere, given the right conditions. The answer to the question of whether this part of the lobes is filled with ionospheric plasma (or not) has significant consequences for our understanding of the near-Earth plasma sheet and ring current.

[4] Plasma measurements in the low-latitude near-Earth lobes have recently been made. The apogee of the Polar satellite has been precessing equatorward at a rate of $15^{\circ}$ per year, and from 2001 on, the apogee has been relatively close to the magnetic equator. Therefore for the last few fall seasons, Polar has passed through this region of interest, crossing the near-Earth nightside plasma sheet at a geocentric distance of $\sim 9 R_{E}$.

[5] Polar has observed a ubiquitous population of cold, streaming ions throughout the near-Earth magnetotail lobes [Moore et al., 2005; T. E. Moore et al., Ionospheric plasmas in the ring current, submitted to Global Physics of the Coupled Inner Magnetosphere, Geophysical Monograph Series, 2005, hereinafter referred to as Moore et al., submitted manuscript, 2005]. This paper will present a statistical survey of these observations of ion outflow streams, showing that these ions are a nearly constant feature of the inner tail region. Occurrence statistics are examined, investigating which geophysical parameters organize the lobal wind occurrence rate. The paper concludes with a discussion of the implications of an ever-present "lobal wind" in the near-Earth tail.

\section{Polar in the Magnetotail}

[6] The Polar satellite was launched in 1996 with an apogee of $9 R_{E}$ geocentric distance above the North Pole [Acuña et al., 1995]. The latitude of apogee has been precessing equatorward, and by 2001 , it was quite close to the magnetic equatorial plane (usually just north of it). Thus the satellite was located in the near-Earth nightside magnetotail lobes for extended periods each orbit.

[7] Throughout the year, the apogee rotates through local time, reaching midnight in late September. A study of the nightside tail region, therefore, should focus on the second half of the year. Figure 1 shows the Polar orbit in SM coordinates on 1 July and 31 December 2001 (projections in the $x-y$ and $y-z$ planes are shown). The latitudinal precession is slow; therefore all of the orbits in this 6-month window are very similar to these two "extremes" shown here. It is seen that the northern hemispheric portion of the orbit has better coverage of the lobe, especially in the beginning months of the window. As will be shown below, there is sufficient coverage of both northern and southern lobe regions to ensure significance of the statistics. Given the slight hemispheric asymmetry, the results will be examined by half-orbit, split at the plasma sheet crossing.

[8] Note that this year, with its asymmetric orbit geometry, was purposefully chosen for this survey. For a given absolute value of magnetic latitude, the southern hemisphere is sampled at a smaller geocentric distance than the northern hemisphere. Therefore the similarities and differences between the southern and northern occurrence statistics can be considered indicative of radial distance effects rather than hemispheric influences. Thus more information is gained from this data set than, say, from the fall 2002 nightside apogee orbits, where the hemispheric asymmetry is much smaller. In the fall of 2003, the satellite spin axis was changed to the ecliptic normal direction, which negatively impacts the viewing of lobal winds. Therefore it is felt that the fall of 2001 is the best season to survey this feature in the Polar data.

[9] Polar made hundreds of orbits in this 6-month window. Excluding the eclipse period from 14 September to 7 October (when the instruments were turned off), the satellite made 208 orbits. Thus there are 416 half-orbits for consideration.

[10] The instrument of relevance on the Polar spacecraft is the Thermal Ion Dynamics Experiment (TIDE) [Moore et al., 1995]. TIDE was designed to obtain mass-resolved measurements of the three-dimensional (3-D0 velocity distribution of the low-energy ions in the magnetosphere, especially the very low densities above the polar regions. TIDE detects the plasma through seven independent energy analyzers that combine electrostatic mirrors with retarding potential analyzers. The seven apertures are arranged in a fan to resolve polar angle with respect to the spacecraft spin axis, while the spin sweeps this fan through azimuth angle. This yields a nearly complete $3-\mathrm{D}$ view. The analyzers can detect particles from protons to molecular ions up to energies of $300 \mathrm{eV}$.

[11] In late 1996, instrument degradation of the start pulse signal led to the loss of mass and polar angle resolution, but TIDE continues to operate with collection of only the stop pulse signals. This "stops" data is what will be analyzed in this study. The loss of mass resolution only affects the calculation of moments from the velocity distributions. The two primary species of interest for this study are $\mathrm{H}^{+}$and $\mathrm{O}^{+}$. It is unclear which of these species dominates the measurements presented below. This ambiguity results in an extra factor of up to 4, if one species is assumed and the ions are actually $100 \%$ of the other species. The loss of polar angle resolution results in a "spreading" of the distribution when attempting to convert spin angle to pitch angle. As seen below, the observation of interest is often very narrow in spin angle, centered about the magnetic field direction. The polar angle summation within TIDE implies that the real distribution is even more tightly confined about the magnetic field.

[12] The results presented below focus on the occurrence statistics of streaming, cold ions as seen by TIDE in the nightside lobes. Therefore the analysis (and the conclusions drawn from them) is unaffected by either of the issues pertaining to the loss of the "starts" information. A forthcoming paper will examine the moments statistics of this population. The influence of stops-only data on the results is relevant to that study, and will be addressed in detail therein.

\section{Case Studies}

[13] Figure 2 presents four half-orbit passes of TIDE data. Roughly 9 hours of data are presented in each summary plot. 

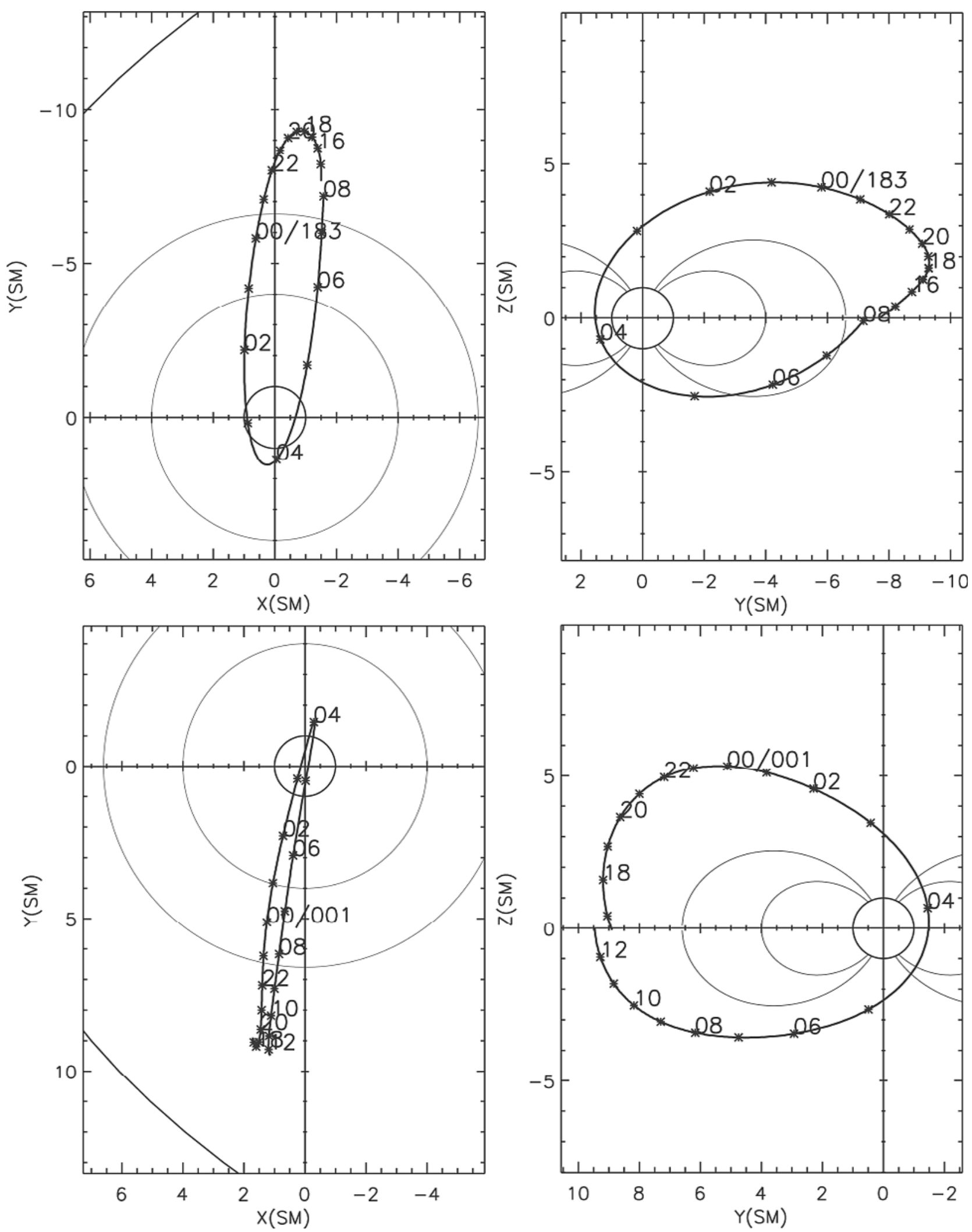

Figure 1. Polar satellite orbits in SM coordinates on 1 July (top) and 31 December (bottom) in the $x-y$ plane (left column) and the y-z plane (right column). The orbit trajectories start and end near apogee at the SM equatorial plane. 

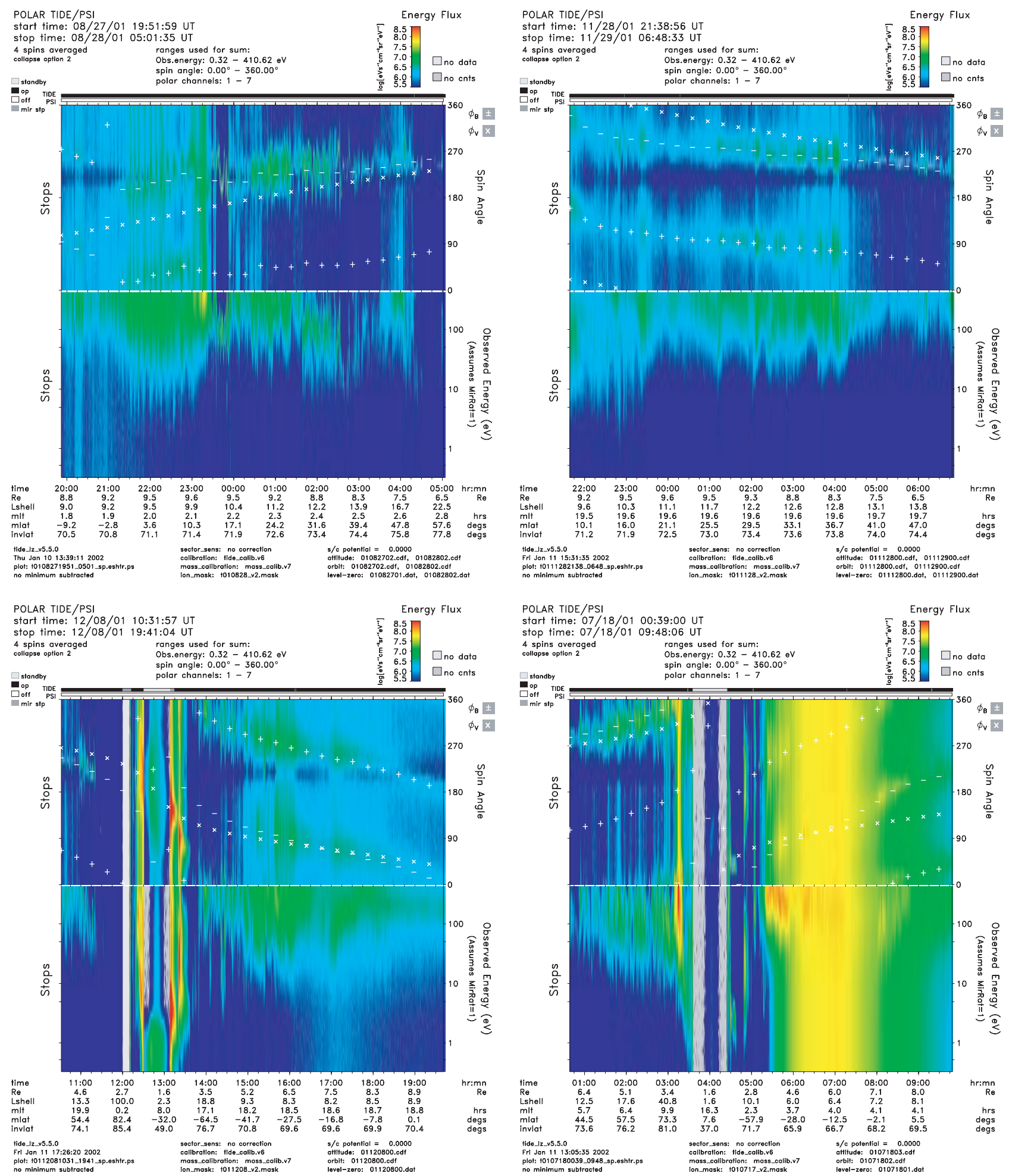

Figure 2. Example half-orbit summary plots of lobal wind observations, on 27 August (upper left), 12 November (upper right), 20 December (lower left), and 18 July (lower right). The upper part of each summary plot shows spin angle distributions while the bottom part shows energy spectra. Ephemeris information is listed along the bottom of each summary plot.

Shown are stops spectrograms of ion energy flux (3-decade logarithmic color scale from blue to red), with spin angle distributions in the upper part and energy distributions in the lower part. The upper two half-orbits show typical northern hemisphere observations of lobal wind, while the lower two half-orbits are typical southern hemisphere spectrograms. Three symbols run through the spin angle spectrogram panel of each summary plot. The cross symbol indicates the spin 
angle containing the ram direction of the spacecraft, while the plus and minus symbols denote the spin angles containing the positive and negative magnetic field directions. Therefore data coincident with the plus sign are of south-to-north flows along the field line, and data at the minus sign spin angle are of north-to-south field-aligned flows.

[14] The upper row of summary plots in Figure 2 shows typical observations of lobal winds. The lobal winds are those populations confined near the magnetic field spin angles. These beams of plasma have much higher flow speeds than their parallel or perpendicular thermal speeds, making the signatures obvious in the spectrograms. Bidirectional streams are often seen near the magnetic equator and unidirectional streams at higher magnetic latitudes. Occasionally, a warm, isotropic, stationary plasma dominates the spectrograms for brief intervals. This is actually a separate population from the streaming lobal winds, which are still present but hard to distinguish when the isotropic plasma intensifies. The upper left half-orbit has a rapid transition between the southern and northern lobes (i.e., this orbit passes through a thin plasma sheet). The upper righthand half-orbit shows a very gradual reversal of the field direction relative to spin angle, indicating a thick plasma sheet at this time. Most northern passes are like these two examples, with clear lobal wind observations. The only unusable northern hemisphere half-orbits are those when background radiation floods the instrument. For the northern lobe observations, contamination is typically from a solar energetic particle event.

[15] The lower row of summary plots in Figure 2 shows one "good" and one "bad" example of southern hemispheric data. The dayside perigee portion of the orbit is located around 1300 UT in the lower left plot and around 0400 UT in the lower right-hand plot. The lower left plot has a low level of penetrating radiation signal, and bidirectional streams are clearly seen from 1500 UT onward. A unidirectional stream is also evident between 1400 and $1500 \mathrm{UT}$, and the transition from bi- to unidirectional wind at higher latitudes is a common feature of the southern hemispheric data (as in the northern hemisphere). In contrast, the lower-right plot has a high level of radiation beltinduced noise in the spectrograms, and no lobal wind measurement can be identified between 0600 and 0700 UT. Even with this contamination, bidirectional streams are evident before and after this interval. Sometimes the noise dominates the TIDE data throughout the entire southern hemisphere passage, and that half-orbit is unusable in the survey.

[16] Note that these streams are different from the hot unidirectional or bidirectional streams seen in the plasma sheet boundary layer [e.g., Williams, 1981; Eastman et al., 1984; Parks et al., 1998] but rather are like the low-energy streams seen by Sharp et al. [1981, 1982] and Candidi et al. [1982] throughout the lobes and plasma sheet. Orsini et al. [1984] found that these tailward flowing low-energy streams can sometimes be converted into the earthward flowing high-energy streams in the plasma sheet boundary layer. This study of Polar TIDE data is therefore complementary to the survey of Sharp et al. [1982], sampling at distances closer to the Earth and across a wide range of magnetic latitudes.
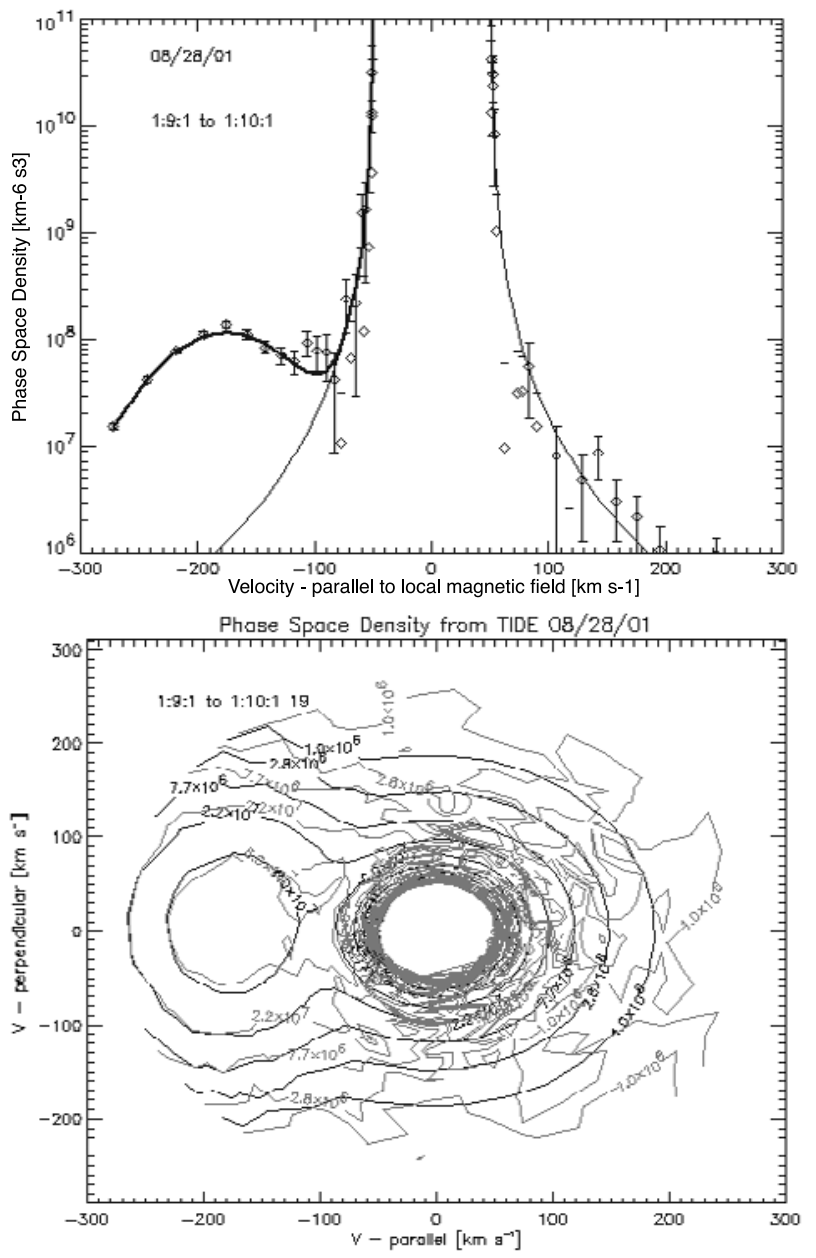

Figure 3. Phase space densities for a typical unidirectional lobal wind measurement (a 1-min average starting at 0109 UT on 28 August, from the upper left part of Figure 2). The top part shows a cut at $v_{\perp}=0$ (i.e., parallel and antiparallel directions) as data points with error bars, with the gray curves indicating the 1 count level. The lower part shows a two-dimensional (2-D) contour plot of the distribution (gray curves). A Maxwellian fit to the data is overlaid (dark solid curves) in both the upper and lower parts.

[17] While a full examination of lobal wind moments will not be provided here, it is useful to calculate the bulk quantities for a good example. Figure 3 shows a phase space density line plot and contour plot of a typical unidirectional lobal wind measurement on 28 August 2001 (as seen in the upper left part of Figure 2). The data was manually fit with a drifting bi-Maxwellian function in order to quantify the distribution. The fit parameters are as follows: a density of $0.19 \mathrm{~cm}^{-3}$, a parallel velocity of $-175 \mathrm{~km} \mathrm{~s}^{-1}$, perpendicular velocities of 5 and $-30 \mathrm{~km} \mathrm{~s}^{-1}$ in the $\mathrm{y}$ and $\mathrm{z}$ directions, and parallel and perpendicular temperatures of $20 \mathrm{eV}$ and $50 \mathrm{eV}$, respectively. Note that these values assume the ions are protons. If the beam is oxygen ions, then the density will be 4 times larger and the velocities will be 4 times smaller.

[18] To continue this illustration, Figure 4 shows moments values of this unidirectional wind from 0000 UT 


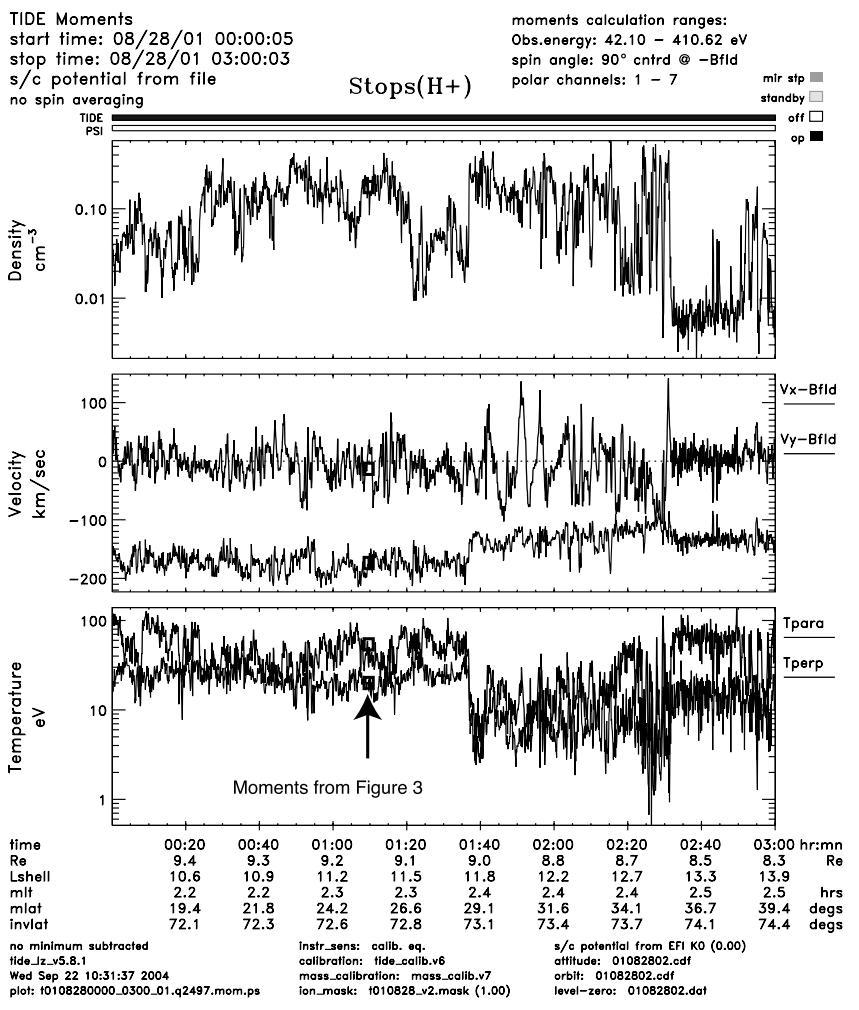

Figure 4. Moments for the unidirectional stream of the lobal wind on 28 August, assuming $\mathrm{H}^{+}$. Shown are density, field-aligned and perpendicular flow speed, and fieldaligned and perpendicular temperature in the three rows, respectively. The velocity space domain of the integration was 50 to $410 \mathrm{eV}$ and a $90^{\circ}$ track width following the negative magnetic field direction. For comparison, the manual-fit moment values from the distribution shown in Figure 3 are overlaid on the numerical moment values as small boxes.

to 0300 UT on 28 August 2001. The values are computed assuming $\mathrm{H}^{+}$. The set of plots presents the density, velocity components, and temperature components in the three parts, respectively. To limit the background contribution, these moments were calculated using integrations over a $90^{\circ}$ track width surrounding the negative magnetic field direction and a $42 \mathrm{eV}$ lower energy limit. This lower energy limit is the closest energy channel boundary below $100 \mathrm{~km} \mathrm{~s}^{-1}$, which is the velocity at which the phase space distribution diverges from the background level, as seen in Figure 3. We believe that this lower energy limit is therefore justified in these moments calculations for this example case study.

[19] It is seen that the values are quite similar to the Maxwellian fit values reported above. These plots punctuate the highly field-aligned nature of the streams, with typical field-aligned flow speeds of $100-200(25-50) \mathrm{km} \mathrm{s}^{-1}$ for $\mathrm{H}^{+}\left(\mathrm{O}^{+}\right)$and temperatures of $5-50 \mathrm{eV}$.

[20] Figures 3 and 4 represent completely independent methods of deriving the bulk parameters of the lobal wind population. The first approach is a trial-and-error Maxwellian fit to the data, comparing the contours by eye and adjusting the values by hand. The second approach is a computational integration of the data within some region of velocity space. The values from the manual fit are shown on the numerically calculated values in Figure 4. That they agree so well offers confidence that these moments calculations are reasonable, and also validates the restricted velocity space region over which we performed the automated numerical integration. A more detailed examination of the moments throughout the selected 6-month nightside apogee period will be conducted in the near future.

\section{Statistical Survey}

[21] With these typical values in mind, a survey of the occurrence of lobal wind observations was conducted for the July-through-December 2001 interval. The survey was by manual identification of the lobal wind from the TIDE summary plots. First, the details of the survey methodology are described. This is followed by an objective presentation of the survey results. Interpretation and discussion is given in the next section.

[22] In the 6-month survey period, there were 416 noneclipse half-orbits. A high-latitude cutoff was applied at $\pm 60^{\circ}$ magnetic latitude for each half-orbit. If the lobal wind data clearly continued beyond this latitude, the pass endpoint was extended to the end of this observation. Similarly, if the TIDE data is unusable (either nonexistent or contaminated), then the endpoint was shifted equatorward until usable TIDE data was found. The cutoff between southern and northern half-orbits was selected at the time when the spin angle containing the magnetic field direction had shifted half way between the average southern lobe angle to the average northern lobe angle. Sometimes this transition was fast, occurring in less than an hour, in which case the adjacent half-orbits were designated as "thin plasma sheet" passes. Otherwise, the adjacent half-orbits were labeled "thick plasma sheet" passes. Some passes were completely unusable because either TIDE was turned off or background radiation swamped the signal. This eliminated 73 half-orbits from the database (23 northern and 50 southern passes), reducing the number of half-orbits in the survey to 343 . The total time included in the survey was 2211.5 hours.

[23] The TIDE summary plots were examined to determine the existence (or not) of lobal winds along each pass. A threshold energy flux value of either $10^{6} \mathrm{eV}\left(\mathrm{s} \mathrm{cm}^{2} \mathrm{sr}\right.$ $\mathrm{eV})^{-1}$ or 5 times greater than the background level (whichever was greater) was used. In addition, the flux peak had to be centered on the magnetic field (along either or both directions) with a maximum-to-minimum ratio in the spin angle distribution of at least a factor of 3 . In the energy spectrograms, the lobal wind flux peak had to be centered at or above $50 \mathrm{eV}$. This energy criterion does not mean that the entire distribution must be above 50, only the energy flux peak. A thorough examination of the appropriate lowerenergy cutoff for moments calculations is deferred to a future study.

[24] Data was considered in 15-min intervals, assigning each window one of three designations: no lobal wind, a unidirectional stream, or bidirectional streams. The magnetic local time of the north-south half-orbit transition was also recorded, along with the most poleward magnetic latitude of bidirectional streams.

[25] Several geophysical and solar wind quantities were averaged over each half-orbit interval and included in the 
survey. These were Dst, total change in Dst, solar wind density and velocity (x-component in GSM coordinates), and the interplanetary magnetic field (IMF) vector ( $\mathrm{y}$ and $\mathrm{z}$ components in GSM coordinates). From these, several other quantities were derived for each half-orbit: rate of change of Dst, solar wind dynamic pressure, total IMF strength (modulus of the $\mathrm{y}$ and $\mathrm{z}$ components), IMF clock angle, solar wind motional electric field $\mathrm{E}_{\mathrm{y}}$, and the Kan-Lee electric field $\mathrm{E}_{\mathrm{K}-\mathrm{L}}$ [Kan and Lee, 1979]. Level 2, hourly averaged data from the Advanced Composition Explorer (ACE) mission were used for solar wind and IMF quantities, and a standard 1-hour shift was applied to account for propagation from the spacecraft to Earth. This crude time shift is felt to be justified because the half-orbits are several hours long, and a few minutes error in the start and end times of the solar wind and IMF averages introduces only a small error.

\subsection{Average Values}

[26] Figure 5 presents occurrence percentages of unidirectional and bidirectional cold ion streams as a function of hours and half-orbit passes. In each part, five columns show various subsets of the data. These are, from left to right: all half-orbit passes, only the northern hemisphere half-orbits, only the southern hemisphere half-orbits, only those passes with thick plasma sheets, and only those passes with thin plasma sheets. The total number of half-orbits (hours) in each of these categories is, respectively, 343 (2211.5), 185 (1483.25), 158 (728.25), 280 (1791.75), and 63 (419.75).

[27] In the top part of Figure 5, the columns are divided into three shades: black denotes the bidirectional stream intervals, gray shows the unidirectional stream intervals, and white is the time when no lobal winds were observed. The percentages above each column give the time when either type of lobal wind was seen relative to the total time for that half-orbit category. All of the percentages are slightly above the two-thirds mark. The total time of the bidirectional lobal wind is higher than that for the unidirectional lobal wind in three of the five categories.

[28] The rest of Figure 5 shows the numbers of half-orbits with unidirectional wind (middle part) and bidirectional wind (lower part), regardless of the length of time they were observed on that orbit. All 10 of the percentages listed here are above $50 \%$, with some above $90 \%$. The two percentages for any one category add up to more than $100 \%$ because many orbits contain both bidirectional and unidirectional winds, as seen in the examples presented in Figure 2. In fact, every single half-orbit with usable data (i.e., all 343) had at least one 15-min interval of lobal wind observation. The bidirectional wind percentages are higher than the unidirectional wind values for all categories except the thin plasma sheet group.

[29] A few average values for the half-orbits in each of the five subset categories are presented in Figure 6. The top part shows the maximum magnetic latitude of bidirectional wind observation. The average latitude for all passes is $25^{\circ}$. This value is slightly higher for southern half-orbits and for thick plasma sheet passes, while it is slightly lower for the other two subsets. The thin plasma sheet passes had the lowest average magnetic latitude of bidirectional to unidirectional wind transition at $17^{\circ}$. Note that only 269 halforbits had a clear upper limit to the bidirectional stream

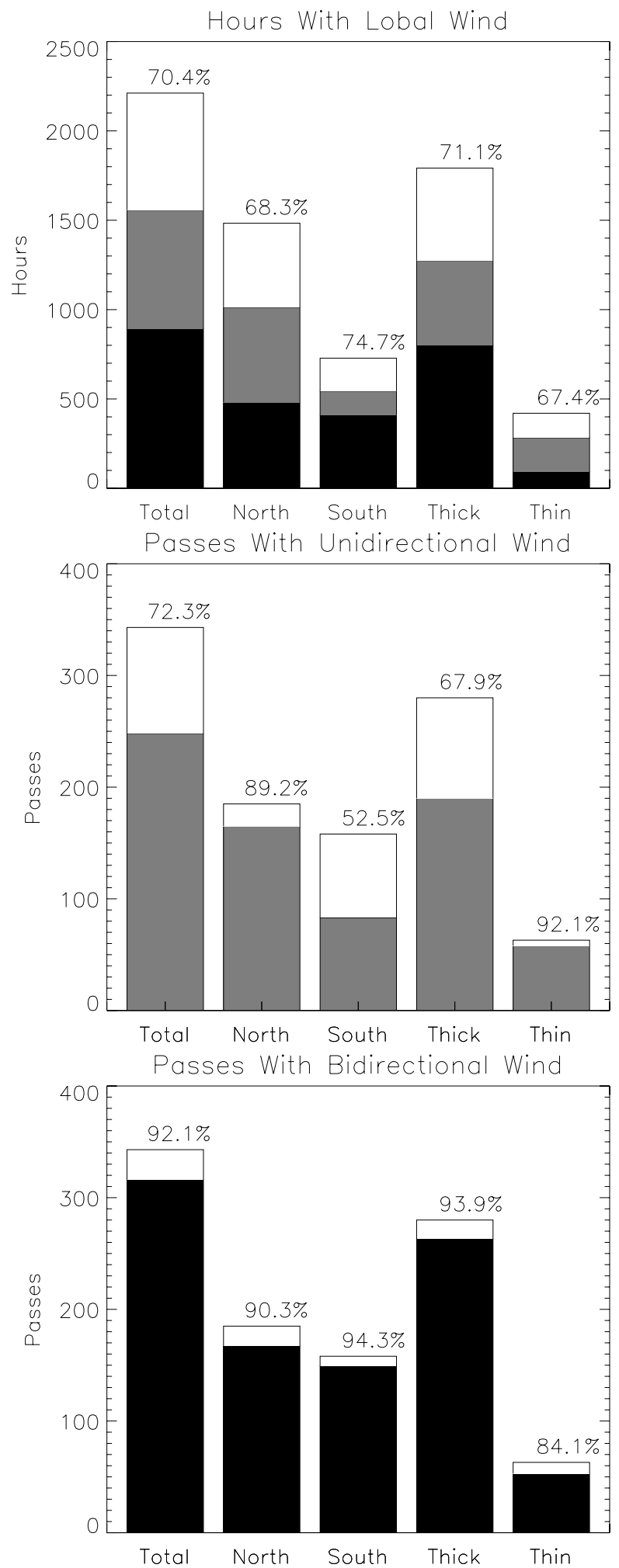

Figure 5. Total number of hours (top) and half-orbits (bottom) in which lobal winds, either unidirectional (gray) or bidirectional (black), were observed (as well as no observation, in white), along with percentages of the total number of hours or half-orbits. The five columns in each chart give the numbers for all half-orbits, the northern halforbits, the southern half-orbits, the half-orbits adjacent to a thick plasma sheet crossing, and the half-orbits adjacent to a thin plasma sheet crossing. 


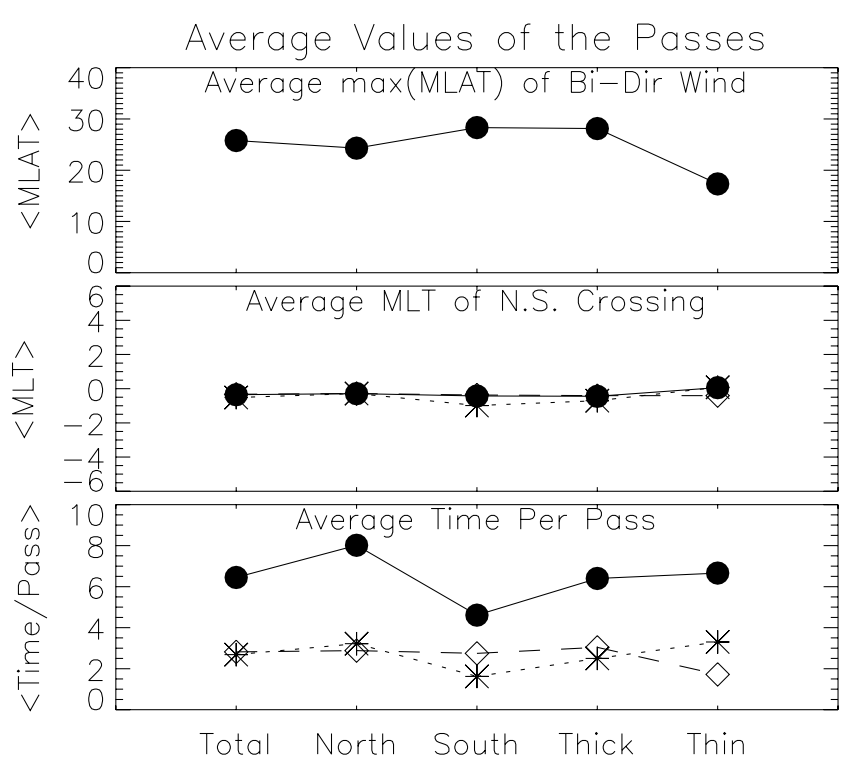

Figure 6. Average values for the half-orbits: (top) maximum magnetic latitude of bidirectional lobal wind observation; (middle) magnetic local time of the plasma sheet crossing; and (bottom) average time per half-orbit in hours. The five columns are as defined in Figure 5. The three lines in the bottom two parts are as follows: solid lines with filled circles are values for all half-orbits in that category; dotted lines with asterisks are values for only those half-orbits that contain unidirectional wind observations; and the dashed lines with open diamonds are values for only those half-orbits that contain bidirectional wind observations.

measurement. If the latitudinal extent of the bi-directional wind was not obvious, it was assigned a fill value and omitted from this average.

[30] The middle parts of Figure 6 presents the average magnetic local time of the plasma sheet crossing. The value for all five categories is within half an hour of midnight. This confirms that the survey database contains a fairly uniform distribution of local times for each category, and the results should not be biased by local time-dependent influences.

[31] The lower part of Figure 6 gives the average time per pass for each category. The three line-symbol combinations are for the total half-orbit duration (in hours) for that category (solid line), unidirectional winds (dotted line), and bidirectional winds (dashed line). It is seen that the southern passes are, in general, shorter than the northern passes, as expected from the slightly asymmetric orbit of Polar in late 2001. Overall, the two types of wind are observed for roughly equal lengths of time per half-orbit, with the notable exception that southern passes tend to have more bidirectional streams while thin plasma sheet passes tend to have more uni-directional streams.

[32] The average values of the geophysical and solar wind parameters for each category are presented in Figure 7 . The format is the same as that in Figure 6. The three line-symbol combinations in each panel show the average values for all passes in that category (solid line), only those passes that contained unidirectional wind observations (dotted line), and only those that contained bidirectional wind data (dashed line).

[33] The first three parts of Figure 7 are the results for Dst. The average Dst was $-19 \mathrm{nT}$, which is a typical quiettime offset to this index [Burton et al., 1975]. Most of the other subsets had a similar average Dst, with the exception being the thin plasma sheet passes, which had a value of $-29 \mathrm{nT}$. Another exception was the southern half-orbits with unidirectional wind, when the average Dst was $-25 \mathrm{nT}$. The average change in Dst during each half-orbit was essentially zero for all categories except the thin plasma sheet passes, when then average change in Dst was $-7.0 \mathrm{nT}$. Similarly, division by the half-orbit duration yields a rate of change in Dst of essentially zero for all categories but the thin sheet passes. The average $\mathrm{d} D s t / \mathrm{dt}$ for that category is $-1.3 \mathrm{nT} / \mathrm{h}$ (all passes), being $-1.6 \mathrm{nT} / \mathrm{h}$ for the unidirectional wind passes and $-1.1 \mathrm{nT} / \mathrm{h}$ for the passes with bidirectional wind.

[34] The next three part in Figure 7 show the average solar wind values. Note that only 310 of the half-orbits had solar wind data available for some portion of its duration. Those without were assigned a fill value and omitted from these averages. The average solar wind density, velocity, and dynamic pressure are $6.2 \mathrm{~cm}^{-3}, 423 \mathrm{~km} \mathrm{~s}^{-1}$, and $1.8 \mathrm{nPa}$, respectively. In general, the subsets have very similar values, again except for the thin plasma sheet passes, where the average values are significantly higher: $8.9 \mathrm{~cm}^{-3}$, $433 \mathrm{~km} \mathrm{~s}^{-1}$, and $3.2 \mathrm{nPa}$.

[35] The average IMF values are given in the next three parts of Figure 7. No orbits were excluded because of missing IMF data. The average values for $B_{y}, B_{z}$, and $B_{\text {total }}$ are $0.50,-0.11$, and $4.1 \mathrm{nT}$, respectively. As before, all of the subsets have roughly the same values. However, the average $B_{y}\left(B_{z}\right)$ for half-orbits with unidirectional wind are systematically more positive (negative) than for all of the passes (roughly $0.2 \mathrm{nT}$ higher in $B_{y}$ and $-0.3 \mathrm{nT}$ lower in $B_{z}$ ). The thin plasma sheet passes also have different (larger in magnitude) average values of $0.83,-0.55$, and $5.3 \mathrm{nT}$ for the three quantities.

[36] The final set of three parts in Figure 7 show the average solar wind electric quantities, specifically $E_{y}$, clock angle, and $\mathrm{E}_{\mathrm{K}-\mathrm{L}}$. Again, it is seen that the unidirectional wind passes have systematically larger values than the averages for all passes in a subset category, and that the values for the thin plasma sheet passes are also systematically larger than the other subset values.

\subsection{Systematic Trends}

[37] The previous subsection presented averages for the various subsets of the TIDE lobal wind database. Here, occurrence characteristics of the lobal wind are compared against the geophysical and solar wind quantities for each orbit in a scatterplot format. While the results presented above can be used for identifying differences in the parameter averages between the categories, the results presented in this subsection can be used to identify which parameters control the variability of these occurrence statistics.

[38] Four occurrence parameters will be considered here. The first is the maximum magnetic latitude of bi-directional lobal wind observation. The other three values are the fraction of the half-orbit with (a) any kind of lobal wind, 

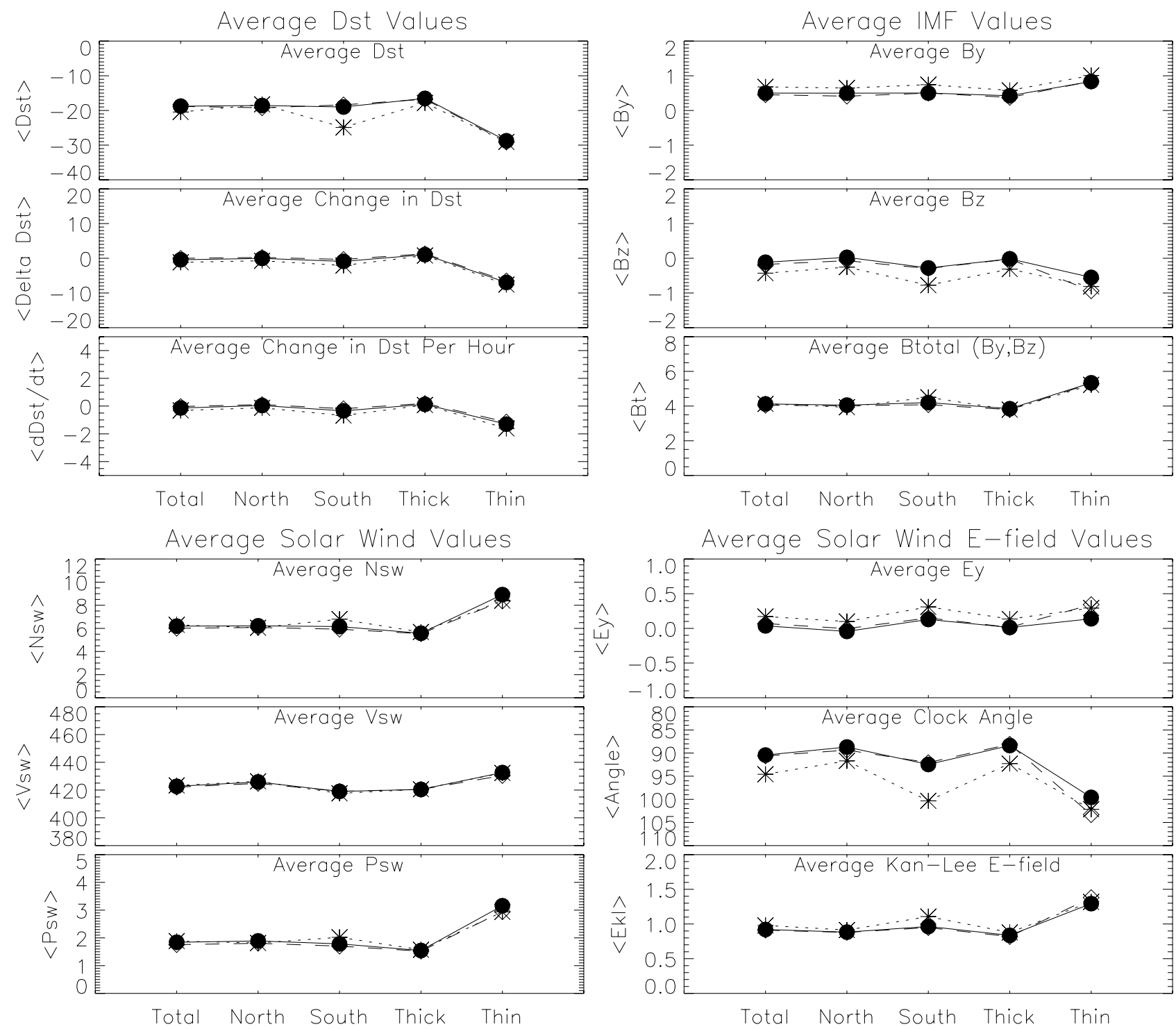

Figure 7. Average geophysical and solar wind values over the half-orbit for the lobal wind observations. The five columns are the same as those in Figure 5. The three lines are the same as those in Figure 6. In the left column, the six rows show (top to bottom) Dst, change in Dst, Dst change per hour, solar wind density, solar wind velocity, and solar wind dynamic pressure. In the right column of each part, the six rows show (top to bottom) IMF $B_{y}$, IMF $B_{z}$, IMF $B_{\text {total }}\left(B_{y}\right.$ and $B_{z}$ components), solar wind $E_{y}$, IMF clock angle, and solar wind Kan-Lee electric field.

(b) unidirectional lobal wind, and (c) bidirectional lobal wind.

[39] Figure 8 shows scatterplots for the four occurrence statistics as a function of Dst. This is a useful parameter to consider because there is anecdotal and statistical evidence that ionospheric ion content in the magnetosphere increases with geomagnetic activity [e.g., Lennartsson et al., 1981; Young et al., 1982; Daglis et al., 1993; Pulkkinen et al., 2001]. Listed on each panel is the Pearson linear correlation coefficient $\left(R_{P}\right)$ and the Spearman rank correlation coefficient $\left(R_{S}\right)$. The solid line through each scatterplot is the bestfit linear regression result, to help see the trend in the data. The dashed line shows the average value in a series of bins across the Dst domain (as another measure of the trend in the data). The number of data points is different for each part, therefore the $95 \%$ confidence level for these correlation coefficients varies between $\sim 0.1$ ( $>200$ data points) and $\sim 0.3$ ( $<50$ data points). Correlations that are "significant" are printed in bold (significant being defined as a $<5 \%$ probability that the coefficient could be this high from two uncorrelated data sets). Over half of the coefficients are significant, indicating that the occurrence frequency of lobal wind is probably correlated with Dst. The significant correlations are both positive for the plot of maximum magnetic latitude for the bidirectional wind (Figure 8a), the plot of wind fraction for any type of wind (Figure 8b), and the plot of bidirectional wind fraction (Figure 8d). However, the correlations for the plot of unidirectional wind fraction (Figure 8c) are negative (and not significant). A positive correlation indicates that the occurrence parameter 


\section{Half-Orbit Fraction Versus Dst}
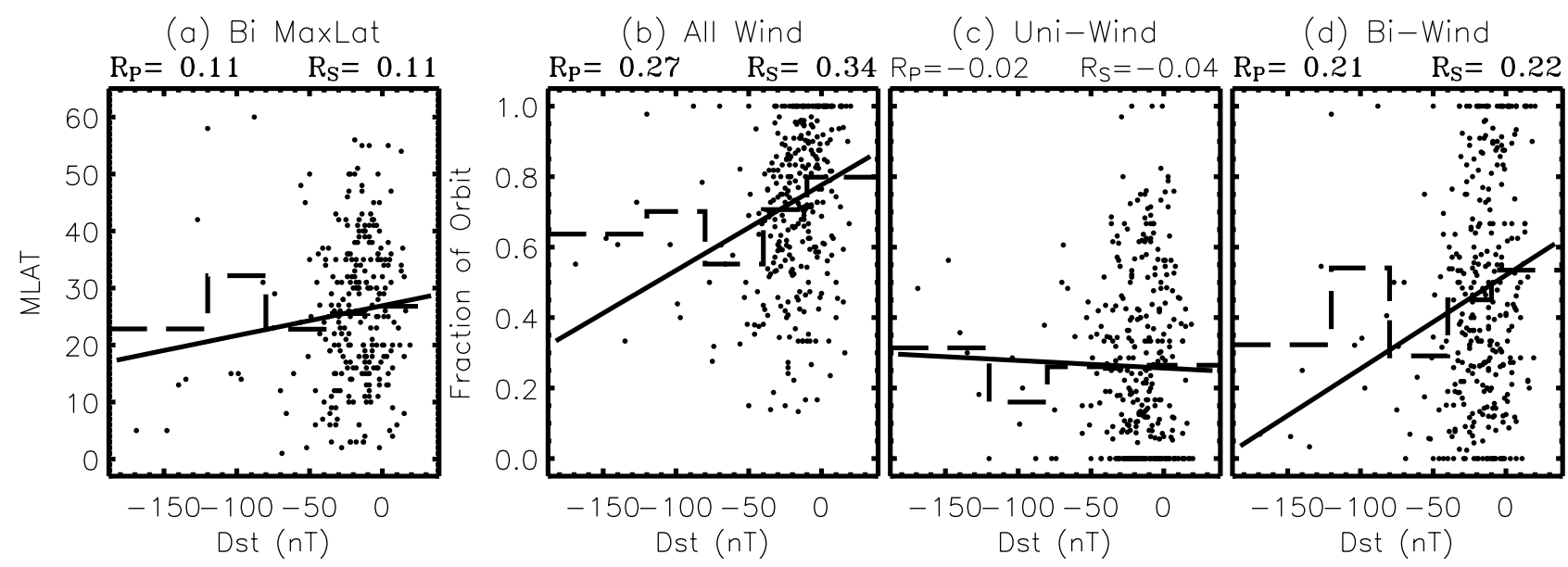

Figure 8. Scatterplots versus the average Dst during the half-orbit for several quantities: (a) maximum magnetic latitude of bidirectional wind observation; (b) fraction of orbit with either kind of lobal wind; (c) fraction of orbit with only unidirectional lobal wind; and (d) fraction of orbit with only bidirectional lobal wind. All of the passes are included in these plots. Listed on each panel is the Pearson linear correlation coefficient $\left(R_{P}\right)$ and the Spearman rank correlation coefficient $\left(R_{S}\right)$. The solid line through each scatterplot is the best-fit linear regression result, and the dashed line is a histogram of the bin averages across the Dst domain.

decreases as Dst goes more negative (i.e., during active times), while a negative value means the parameter increases with geomagnetic activity. Therefore the trend, albeit a very weak one, is that the bidirectional winds decrease in frequency during magnetic storms while the unidirectional wind frequency is unchanged.

[40] Figure 9 is another set of scatterplots, this time against the average Kan-Lee electric field value for each half-orbit. This parameter is a good measure of the coupling function between the solar wind and the ionosphere, and is a useful quantity because outflow has been related to Poynting flux into the ionosphere [e.g., Strangeway et al., 2000]. As with Figure 8, many of the coefficients on this plot are significant. The maximum magnetic latitude of bidirectional wind is negatively correlated with $\mathrm{E}_{\mathrm{K}-\mathrm{L}}$, meaning that this latitude decreases with increasing energy transfer to the magnetosphere. Similarly, the occurrence rate of bidirectional wind is also negatively correlated. Unidirectional wind fraction is positively correlated. However, the orbit fraction of any kind of wind is not significantly correlated with $\mathrm{E}_{\mathrm{K}-\mathrm{L}}$. This implies that the type of wind changes with $\mathrm{E}_{\mathrm{K}-\mathrm{L}}$, but not the overall amount of lobal wind. As with Dst, however, the trends in the scatterplots are not particularly evident by visual inspection, and the correlations, while statistically significant, are suspect.

[41] Figure 10 shows scatter plots of the occurrence parameters against solar wind dynamic pressure. Dynamic pressure is thought to a controlling factor of ionospheric outflow, particularly on the dayside [e.g., Moore et al., 1999; Cully et al., 2003a]. The trends are the same as for $\mathrm{E}_{\mathrm{K}-\mathrm{L}}$. The maximum magnetic latitude of bi-directional wind is negatively correlated (Figure 10a), indicating that
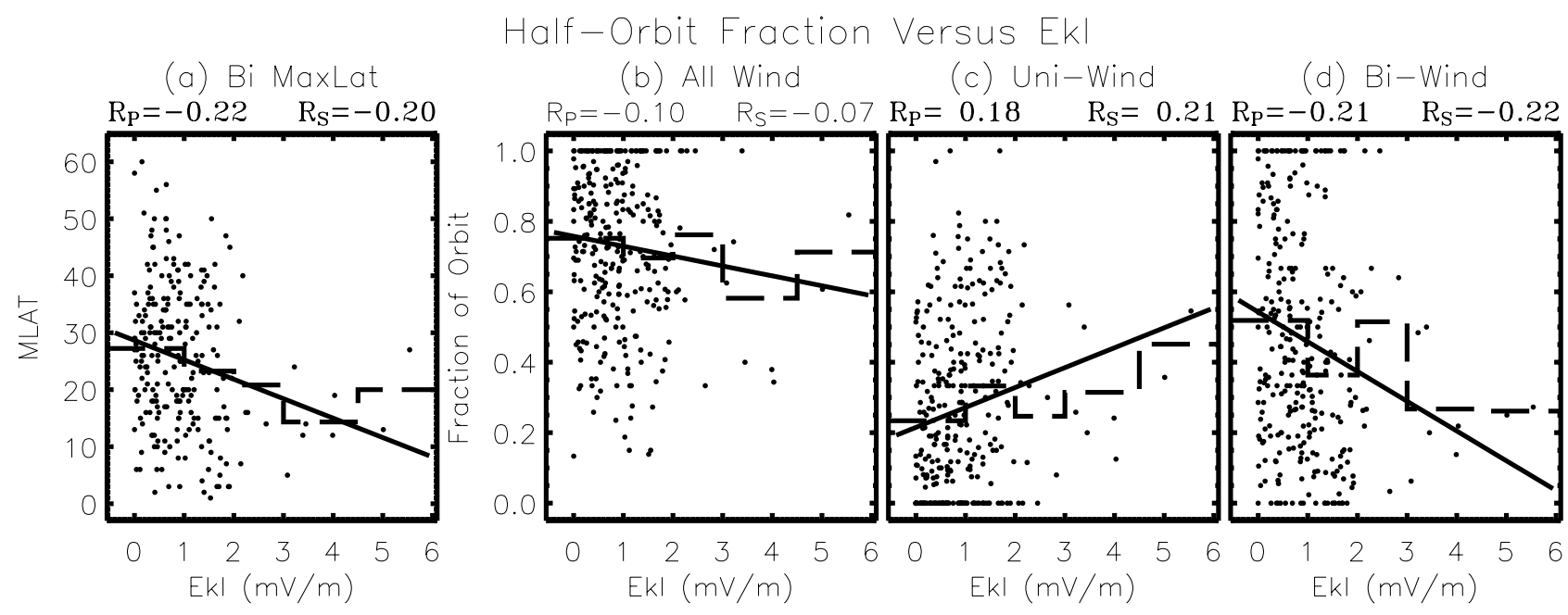

Figure 9. Like Figure 8, except that the x-axis values are Kan-Lee electric fields. 


\section{Half-Orbit Fraction Versus Psw}
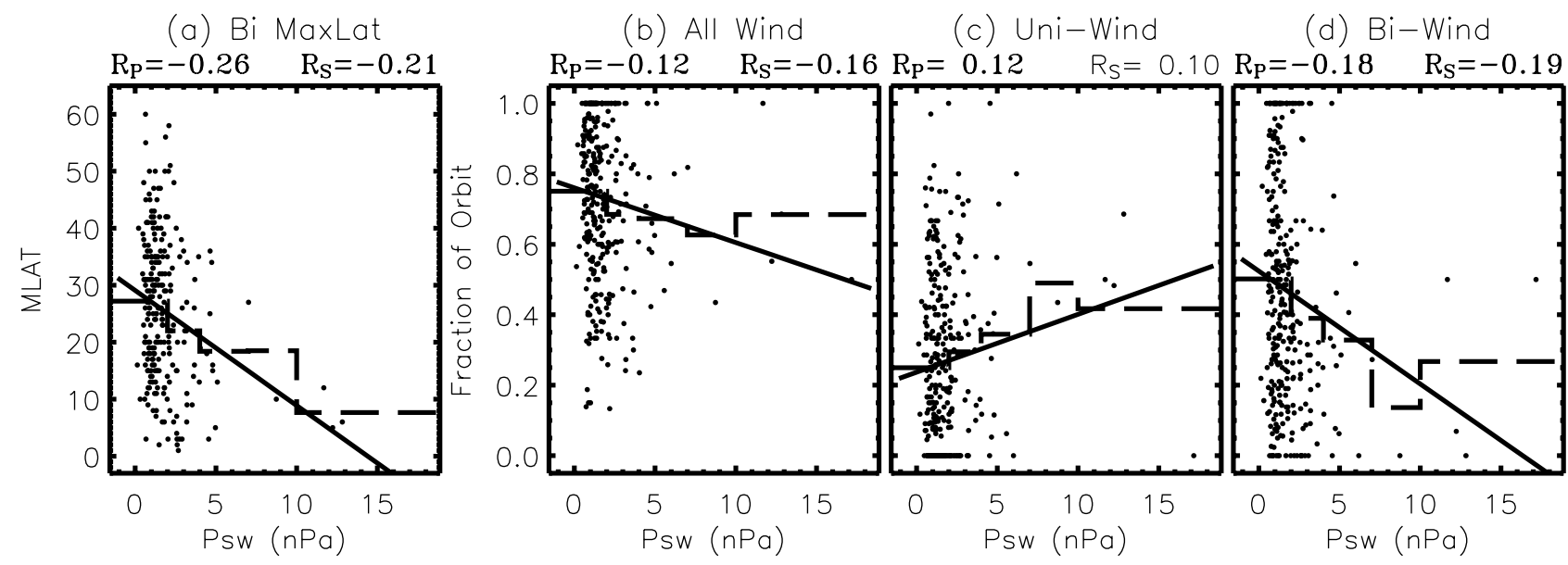

Figure 10. Like Figure 8, except that the $\mathrm{x}$-axis values are solar wind dynamic pressures.

as $\mathrm{P}_{\mathrm{sw}}$ increases, this latitude decreases. A similar trend is seen for the fraction of the half-orbit with bidirectional wind (Figures 10a and 10d); it decreases with increasing $\mathrm{P}_{\mathrm{sw}}$. Unidirectional wind fraction, however, is positively correlated. The correlation of the fraction of any kind of wind with $\mathrm{P}_{\mathrm{SW}}$ is not statistically significant. So, the trend is the same as in Figure 9; as the parameter increases, there is a shift from bidirectional wind to unidirectional wind. However, like Figures 8 and 9, the trends are barely above the significance level and are hard to detect by simply looking at the scatterplots.

[42] These types of plots were also produced for the other solar wind and geophysical parameters mentioned in section 4.1, and also for all five subsets of the database discussed in section 4.1. While these scatterplots will not be shown here, the $R_{P}$ values are listed in Table 1 . As on the plots, the statistically significant coefficients (at the 95\% confidence level) are printed in bold.

[43] A few highlights from Table 1 should be noted. One finding is that the significant correlation coefficients for the change in Dst (both in magnitude over the half-orbit as well as rate) are very similar to those for $\mathrm{E}_{\mathrm{K}-\mathrm{L}}$. The change of Dst is an interesting parameter to consider because it has been argued that the supply of ionospheric material to the inner magnetosphere changes with storm phase [e.g., Daglis et $a l ., 1993]$. Dst decreases during the main phase of magnetic storms and increases (recovers) back toward zero during the recovery phase. As expected, these derivatives of Dst are better related to the energy coupling function than to Dst itself.

[44] Another interesting result is that the solar wind density coefficients exhibit similar significance to the $\mathrm{P}_{\mathrm{sw}}$ coefficients. Solar wind velocity, however, is not nearly as significantly correlated as $\mathrm{n}_{\mathrm{sw}}$ or $\mathrm{P}_{\mathrm{sw}}$. Those that are significant, however, are all positive, indicating that the occurrence parameters increase with increasing $\mathrm{v}_{\mathrm{sw}}$.

[45] It should be mentioned that none of the correlations is significant for IMF $B_{y}$; this parameter is not a controlling factor of lobal wind occurrence. The total IMF magnetic field strength is also not particularly well correlated with the selected lobal wind values. However, IMF $B_{z}$, IMF clock angle, and $\mathrm{E}_{\mathrm{y}}$ have significant correlations that closely resemble the $\mathrm{E}_{\mathrm{K}-\mathrm{L}}$ values and trends.

[46] A final comment about Table 1 is that the correlation coefficients for the thin plasma sheet passes are almost never significant. Only two of the $48 \mathrm{R}_{\mathrm{P}}$ values reach the $95 \%$ confidence level for statistical significance, and even those are not much above the threshold.

\section{Discussion}

\subsection{Parameters Controlling the Occurrence of Lobal Wind}

[47] Section 4 shows that streaming, cold ions are ubiquitous in the near-Earth nightside magnetotail lobes. These lobal winds are present on every half-orbit pass in the 6-month interval of the survey, and most of the half-orbits contain intervals of each type of wind (unidirectional and bidirectional streams). Presented here are interpretations of some of the results presented above.

[48] The trends seen in Figure 5 are indicative of the rest of the results. For instance, more (less) bidirectional (unidirectional) wind is observed on the southern hemispheric half-orbits than on the northern ones. Because the southern passes are in general at smaller radial distances from the Earth (due to orbit geometry), this means that it is easier for the lobal wind stream to traverse the plasma sheet closer to the Earth than it is farther downtail. Figure 6a reflects this with a slightly higher maximum magnetic latitude of bidirectional wind for the southern passes than for the northern passes. This can be explained by the amount of curvature in the magnetic field lines as a function of distance from Earth. It is expected that the curvature is greater (i.e., smaller radius of curvature) at larger distances, as the field transitions from a dipole configuration near the Earth to a stretched tail configuration far away from Earth. In addition, because of the geometric asymmetry, the distance traveled by the northward-bound particles in the northern hemisphere is greater than the southward-bound particles in the southern hemisphere. The larger distance (and therefore time-of-flight) could lead to greater attenuation of the fluxes. 
Table 1. Correlation Coefficients for Lobal Wind Values With Various Parameters

\begin{tabular}{|c|c|c|c|c|c|}
\hline & All Passes & North Passes & South Passes & Thick PS Passes & Thin PS Passes \\
\hline \multicolumn{6}{|c|}{ Correlations With Dst } \\
\hline $\operatorname{Max}($ MLAT) of bidir. wind & 0.11 & 0.10 & 0.16 & -0.02 & 0.34 \\
\hline Orbit fraction, all wind & 0.27 & 0.26 & 0.30 & 0.26 & 0.28 \\
\hline Orbit fraction, unidir. wind & -0.02 & 0.12 & -0.23 & 0.05 & -0.01 \\
\hline Orbit fraction, bidir. wind & 0.21 & 0.12 & 0.36 & 0.15 & 0.24 \\
\hline \multicolumn{6}{|c|}{ Correlations With $\Delta D$ st } \\
\hline Max(MLAT) of bidir. wind & 0.25 & 0.25 & 0.28 & 0.19 & 0.21 \\
\hline Orbit fraction, all wind & 0.06 & 0.14 & -0.01 & 0.10 & -0.11 \\
\hline Orbit fraction, unidir. wind & -0.16 & -0.12 & -0.29 & -0.08 & -0.18 \\
\hline Orbit fraction, bidir. wind & 0.17 & 0.24 & 0.18 & 0.13 & 0.08 \\
\hline \multicolumn{6}{|c|}{ Correlations With dDst/dt } \\
\hline Max(MLAT) of bidir. wind & 0.22 & 0.22 & 0.25 & 0.15 & -0.18 \\
\hline Orbit fraction, all wind & 0.04 & 0.12 & 0.01 & 0.14 & -0.22 \\
\hline Orbit fraction, unidir. wind & -0.16 & -0.12 & -0.29 & -0.05 & -0.23 \\
\hline Orbit fraction, bidir. wind & 0.15 & 0.21 & 0.19 & 0.14 & 0.03 \\
\hline \multicolumn{6}{|c|}{ Correlations With Nsw } \\
\hline Max(MLAT) of bidir. wind & -0.25 & -0.16 & -0.40 & -0.15 & -0.25 \\
\hline Orbit fraction, all wind & $-\mathbf{0 . 1 7}$ & -0.17 & -0.18 & -0.15 & -0.16 \\
\hline Orbit fraction, unidir. wind & 0.05 & -0.12 & 0.27 & -0.04 & -0.03 \\
\hline Orbit fraction, bidir. wind & -0.16 & -0.03 & -0.30 & -0.08 & -0.11 \\
\hline \multicolumn{6}{|c|}{ Correlations With Vsw } \\
\hline Max(MLAT) of bidir. wind & 0.06 & -0.01 & 0.22 & 0.15 & -0.16 \\
\hline Orbit fraction, all wind & 0.12 & 0.15 & 0.11 & 0.16 & -0.04 \\
\hline Orbit fraction, unidir. wind & 0.13 & 0.18 & 0.02 & 0.12 & 0.07 \\
\hline Orbit fraction, bidir. wind & -0.02 & -0.04 & 0.06 & 0.03 & -0.10 \\
\hline \multicolumn{6}{|c|}{ Correlations With Pdyn,sw } \\
\hline Max(MLAT) of bidir. wind & -0.26 & -0.21 & -0.35 & -0.14 & -0.25 \\
\hline Orbit fraction, all wind & -0.12 & -0.12 & -0.13 & -0.10 & -0.12 \\
\hline Orbit fraction, unidir. wind & 0.12 & -0.01 & 0.33 & 0.05 & -0.01 \\
\hline Orbit fraction, bidir. wind & -0.18 & -0.10 & -0.30 & -0.11 & -0.09 \\
\hline \multicolumn{6}{|c|}{ Correlations With IMF By } \\
\hline $\operatorname{Max}($ MLAT) of bidir. wind & -0.09 & -0.07 & -0.12 & -0.07 & -0.06 \\
\hline Orbit fraction, all wind & -0.06 & -0.03 & -0.10 & -0.07 & -0.01 \\
\hline Orbit fraction, unidir. wind & 0.06 & 0.11 & 0.02 & 0.07 & -0.01 \\
\hline Orbit fraction, bidir. wind & -0.09 & -0.13 & -0.08 & -0.10 & 0.00 \\
\hline \multicolumn{6}{|c|}{ Correlations With IMF Bz } \\
\hline Max(MLAT) of bidir. wind & 0.22 & 0.28 & 0.17 & 0.27 & -0.01 \\
\hline Orbit fraction, all wind & 0.10 & 0.11 & 0.12 & 0.16 & -0.11 \\
\hline Orbit fraction, unidir. wind & -0.14 & -0.12 & -0.21 & -0.13 & -0.10 \\
\hline Orbit fraction, bidir. wind & 0.12 & 0.25 & 0.22 & 0.21 & 0.00 \\
\hline \multicolumn{6}{|c|}{ Correlations With IMF Bt } \\
\hline $\operatorname{Max}($ MLAT) of bidir. wind & -0.10 & 0.07 & -0.38 & 0.02 & -0.11 \\
\hline Orbit fraction, all wind & -0.03 & 0.02 & -0.12 & -0.02 & 0.04 \\
\hline Orbit fraction, unidir. wind & 0.07 & 0.00 & 0.25 & 0.01 & 0.01 \\
\hline Orbit fraction, bidir. wind & -0.08 & 0.01 & -0.24 & -0.02 & 0.03 \\
\hline \multicolumn{6}{|c|}{ Correlations With Ey } \\
\hline $\operatorname{Max}($ MLAT) of bidir. wind & -0.26 & -0.32 & -0.18 & -0.32 & -0.03 \\
\hline Orbit fraction, all wind & -0.09 & -0.12 & -0.09 & -0.16 & 0.11 \\
\hline Orbit fraction, unidir. wind & 0.13 & 0.16 & 0.20 & 0.13 & 0.13 \\
\hline Orbit fraction, bidir. wind & -0.17 & -0.25 & -0.19 & -0.21 & -0.03 \\
\hline \multicolumn{6}{|c|}{ Correlations With IMF Clock Angle } \\
\hline $\operatorname{Max}($ MLAT) of bidir. wind & -0.16 & -0.200 & -0.14 & -0.15 & -0.02 \\
\hline Orbit fraction, all wind & -0.08 & -0.08 & -0.11 & -0.11 & 0.11 \\
\hline Orbit fraction, unidir. wind & 0.18 & 0.18 & 0.28 & 0.16 & 0.12 \\
\hline Orbit fraction, bidir. wind & -0.200 & -0.25 & -0.25 & -0.19 & -0.03 \\
\hline \multicolumn{6}{|c|}{ Correlations With $E_{K-L}$} \\
\hline Max(MLAT) of bidir. wind & -0.22 & -0.17 & $-\mathbf{0 . 3 0}$ & -0.16 & -0.11 \\
\hline Orbit fraction, all wind & -0.10 & -0.10 & -0.12 & -0.13 & 0.12 \\
\hline Orbit fraction, unidir. wind & 0.18 & 0.17 & 0.29 & 0.13 & 0.11 \\
\hline Orbit fraction, bidir. wind & -0.21 & -0.25 & -0.27 & -0.19 & 0.00 \\
\hline
\end{tabular}


[49] However, as listed in Table 1, the northern and southern half-orbit correlation coefficients are often very similar. This is illustrated by the fact that they coefficients are nearly always of the same sign when both hemispheres have significant correlations with some parameter. It appears that the two hemispheres are responding similarly to the selected activity monitors; it is just a matter of degree. This lends credence to the hypothesis that the hemispheric differences actually represent radial distance effects.

[50] Another feature in Figure 5 is the differences between thick and thin plasma sheet passes. The greater than 4-to-1 ratio of thick to thin passes in the database is expected because the selected survey interval is during solar maximum. That is, during solar minimum, this ratio would be larger. As activity increases, the plasma sheet thins, the field line radius of curvature decreases, turbulence in the tail increases, and it is harder for the streams to traverse the plasma sheet (and therefore be observed as a bidirectional wind). The plasma is isotropized and/or accelerated in the tail and is no longer a well-defined lobal wind, but rather part of the bulk plasma in the plasma sheet. Therefore the fact that the thin sheet passes are dominated by unidirectional wind observations is reasonable. Even though $84 \%$ of the thin sheet passes have bidirectional flows, the average duration of these flows is short (1.7 hours) relative to the overall bidirectional flow length average (2.8 hours), as seen in Figure 6c. In addition, the average parameter values of the thin current sheet subset relative to other subsets (Figure 7) is indicative of active times versus quiet times.

[51] It is also interesting that the overall percentages of lobal wind occurrence for half-orbits associated with thick and thin plasma sheets are nearly equal $(71 \%$ and $67 \%$, respectively). Therefore the reduction in bidirectional stream observation is matched by a similar increase in unidirectional wind observation. That is, the cold ions are continuing to stream out of the ionosphere and into the nearEarth magnetotail, regardless of activity, but they are less likely to cross through the plasma sheet during active times. Note that this study is making no claims about the total amount of ions emitted from the ionosphere during quiet or active times because we have only examined occurrence frequencies and not the trends in the densities and fluxes of these cold streams.

[52] This trend of a lower high-latitude cut off for the bidirectional streams could also be indicative of a smaller "closed" part of the plasma sheet. That is, this magnetic latitude demarks the location of a near Earth neutral line. In this case, the other stream is either lost to the downstream solar wind or accelerated back toward the Earth in the plasma sheet boundary layer.

[53] Figures 8-10 and Table 1 reveal that the lobal wind occurrence rates during thin plasma sheet passes are essentially uncorrelated with the selected activity parameters. Only Dst seems to be a significant organizer of the maximum latitude of bi-directional wind and the fraction of the orbit with any kind of wind (both decrease with more negative $D s t$ ). Conversely, the thick plasma sheet passes show significant correlations with most of the parameters (all but $B_{y}$ and $\left.B_{\text {total }}\right)$, in general following the trend of less (more) bidirectional (unidirectional) wind observations as activity increases. This is puzzling because thin current sheets are a defining feature of magnetic activity in the magnetotail. The lack of correlation for the thin plasma sheet passes is perhaps because the magnetosphere usually undergoes rapid topological changes during active times, and so averaging the parameters over the entire half-orbit is not a valid assumption for these passes. Additional analysis of what controls the lobal wind occurrence statistics during thin plasma sheet intervals is deferred to a future study.

[54] The difference between bi-directional wind and unidirectional winds seen in Figures 7-10 can also be explained in terms of geomagnetic activity. As activity increases, unidirectional wind observations become more prevalent at the expense of bidirectional wind observations. It is noteworthy that the fraction of half-orbit with any kind of wind is often less correlated with geomagnetic activity than either the unidirectional wind fraction or bidirectional wind fraction. This indicates that the cold ions are produced at all levels of activity, but that they have difficulty crossing the plasma sheet as activity increases. Unidirectional streams begin to dominate. Ions in these unidirectional streams are scattered near the equatorial plane, acting as a source for the plasma sheet rather than stream through it to form a bidirectional flow. Therefore it can be inferred that these streams are supplying ions to the plasma sheet. Keep in mind that every usable orbit had at least one 15-min interval of lobal wind observation; these streams are always flowing through the magnetosphere. Therefore these ions represent a reservoir that instantaneously and continuously feed the energetic populations during active times. That is, prompt signatures of energetic $\mathrm{O}^{+}$in the inner magnetosphere [e.g., Daglis and Axford, 1996; Mitchell et al., 2003] do not need elaborate scenarios to draw out and accelerate the topside ionosphere because a candidate seed population is already present.

[55] The conclusions and interpretations mentioned above are, perhaps, difficult to defend because of the dubious statistical trends in the scatter plots. Even with this disclaimer, the general trends seen in each of the solar wind and geophysical parameters are usually the same (if evident at all): some portion of the counterstreaming ion beams is lost during active times and thus the frequency of bidirectional wind drops while the fraction of unidirectional wind increases. Because all three parameters exhibit the same overall trend, it is thought that these trends are real. Values for the linear fits, however, will not be given because the conclusion is qualitative.

\subsection{Source Region: Review of Modeling Studies}

[56] It is beyond the scope of this paper to definitively address the question of the source region of these cold, streaming ions in the near-Earth nightside lobes. However, a brief overview of the recent modeling efforts to connect the ionospheric and magnetospheric terrestrial origin ion observations is given below.

[57] The most common form of numerical modeling used to address the issue of ionospheric plasma in the magnetosphere is kinetic particle tracking simulations. In these studies, thousands to millions of "particles" are launched from some source region and allowed to convect through space under the influence of a specified magnetic and electric field configuration (static or dynamic). Much has been learned from these efforts. For example, Delcourt et al. [1988a] and Wodnicka and Banaszkiewicz [1997] have 
studied warm $\mathrm{O}^{+}$bouncing through the inner magnetosphere, concluding that it can be a significant (but minor) contributor to the Dst index. The model in the first of these studies has also been extensively used to quantify the flow of ionospheric plasma from the high-latitude ionosphere [e.g., Delcourt et al., 1988b, 1989, 1990, 1994; Chappell et al., 2000; Moore et al., 2000, 2005; Moore et al., submitted manuscript, 2005; Huddleston et al., submitted manuscript, 2005]. Delcourt et al. [1994] performed a large-scale study of ionospheric contributions to the plasma sheet and ring current, finding that very low energy (in the electron volt range) polar cap $\mathrm{H}^{+}$outflow and the dayside cusp region feeds the quiet time plasma sheet, computing plasma sheet densities close to those observed. An interesting result of this study is the conclusion that there is a narrow window of initial energy, pitch angle, and location that ions must have in order to contribute to the tail population. Huddleston et al. (submitted manuscript, 2005) used this model to calculate the fate of Polar TIDE perigee observations of ion outflow. They found that the dayside auroral zone and cusp regions have the most access to the plasma sheet and ring current. Again, simulations showed that the observed outflow fluxes were capable of producing a majority of the observed magnetospheric density. Moore et al. [2005] simulated solar wind entry as well as ionospheric outflow, concluding that the dayside auroral zone can rival the solar wind as the dominant contributor to the near-Earth magnetosphere.

[58] Peroomian and Ashour-Abdalla [1996] performed a robust study of particle simulations from the ionosphere through a static magnetosphere with their kinetic model. They concluded that the ionosphere is capable of producing a plasma sheet of modest density, particularly near the Earth. In addition, Ashour-Abdalla et al. [1997] dissected Geotail velocity distributions into their source regions via time-reversed particle modeling, finding that a significant (but minor) amount of the $\mathrm{H}^{+}$in the tail could originate in the ionosphere. This model has recently been used to study the access of solar wind plasma into the magnetosphere with a time-dependent magnetospheric topology [Peroomian, 2003], finding that solar wind entry can be large but is often quite localized.

[59] A final kinetic modeling study that will be mentioned here is Cully et al. [2003b]. They used Akebono observations of ion outflow as a boundary condition for their particle simulations, concluding that the ionosphere is a preferential supplier of ions to the region of substorm origination.

[60] Another numerical technique is multi-fluid magnetohydrodynamic simulations. Winglee [2000, 2003] pioneered this method, concluding that the geopause (the extent of ionospheric plasma dominance to either the density or pressure) can be quite large and includes much of what is normally considered as the magnetosphere.

[61] It appears that much work has been done to link the ionosphere to the magnetosphere. Another possibility exists, however, and that is the recirculated plasmasphere (the ultimate source then being the midlatitude ionosphere, rather than the auroral or polar cap ionosphere). Freeman et al. [1977] first suggested that the storm time drainage plume of the plasmasphere could convect over the poles and supply the plasma sheet. Elphic et al. [1996] and Borovsky et al. [1997] have elaborated on this theme. There is evidence for plasmaspheric material reaching the magnetopause during active times [e.g., Su et al., 2000]. The fate of these plasmaspheric ions, however, is uncertain. Observations show that the ions can either go into the dayside magnetosheath [e.g., Gosling et al., 1990; Fuselier et al., 1991] or over the polar cap [e.g., Su et al., 2001]. The question of whether these particles reach the nightside plasma sheet is still unresolved.

[62] There does not seem to be a consensus opinion of these previous studies about the dominant source region for ionospheric ions in the magnetosphere. This review was meant as a reminder of the rich history of studies addressing ionospheric ions in the magnetosphere, to present an up-to-date survey of the numerical work in this field, and to reveal that this topic is an open area of research.

\section{Conclusions}

[63] Polar TIDE observations of cold, streaming ions in the nightside magnetotail lobes have been surveyed. The main conclusion is that these streams are common; every single usable orbit in the 6-month window included in the study contained at least one 15-min interval of these lobal winds. Over $70 \%$ of the time in the surveyed database contained lobal winds.

[64] They are often unidirectional streams at the middle to high latitudes, flowing away from the nearest polar ionosphere. Over $70 \%$ of the half-orbit passes in the database contained unidirectional wind observations. Near the equator, bidirectional flows are often seen. Over $90 \%$ of the halforbits contained this type of measurement. The average maximum magnetic latitude for the bidirectional streams was $25^{\circ}$, but this has a large variance, ranging from $3^{\circ}$ to $61^{\circ}$.

[65] The occurrence frequency of unidirection or bidirectional winds depends on geomagnetic activity. The Dst index is one of the better organizing parameters of the data, with lobal wind observations decreasing during storm times. The variance is large, however, and a wide range of occurrence characteristics is found across the activity spectrum. Other parameters that seem to organize the data are the rate of change of Dst, solar wind dynamic pressure, IMF $B_{z}$, and solar wind electric field. Bidirectional streams are interpreted as indicating the minimum size of the closed flux tube region. The reduced frequency of bidirectional streams with activity level times is consistent with the thinning of the plasma sheet during these times.

[66] Most of the solar wind and geophysical parameters considered in this study show the same trends in the occurrence frequencies, lending validity to our general conclusion (even though the individual trends are weak). The general trend among these parameters is that the observance of bi-directional streams decreases with activity level, while the observance of unidirectional streams increases. The occurrence rate of any kind of lobal wind is far less correlated, in general, with activity level. This implies that the streams are ubiquitously present, even during storms and substorms, but they have difficulty traversing the plasma sheet during active times. The high frequency of lobal wind observation suggests that these 
cold, streaming ions are a continuous source of terrestrial origin plasma for the near-Earth plasma sheet.

[67] This finding has real implications. The cold streaming ions are a continuous source of plasma in the near-Earth magnetotail, a reservoir of particles just waiting to be snatched up into the plasma sheet and convected into the inner magnetosphere. There is no need to wait for outflow and circulation through the lobes and distant tail to get an ionospheric contribution to the storm-time ring current. These lobal winds provide an immediately available supply at the initiation of geomagnetic activity.

[68] The role of the solar wind is to be decelerated and supply energy to geospace, while the role of the ionosphere is to be accelerated and absorb this free energy. The results of this study confirm the truth of this statement. During active times, the lobal winds feed the plasma sheet, becoming isotropized, accelerated, and eventually convected into the inner magnetosphere.

[69] Acknowledgments. Funding for this study was provided by NASA under grant NAG5-10887 and NNG04G153G. The authors would like to thank Mike Chandler at NASA MSFC for providing the software used for manually fitting the TIDE velocity space distributions. The authors also thank the Kyoto World Data Center for the Dst data and CDAWeb for the solar wind data from the ACE spacecraft (and D. J. McComas and N. Ness for providing their data to CDAWeb).

[70] Arthur Richmond thanks Masahito Nose and another reviewer for their assistance in evaluating this paper.

\section{References}

Acuña, M. H., K. W. Ogilvie, D. N. Baker, S. A. Curtis, D. H. Fairfield, and W. H. Mish (1995), The Global Geospace Science Program and its investigations, in The Global Geospace Mission, edited by C. T. Russell, p. 5-21, Springer, New York.

Ashour-Abdalla, M., et al. (1997), Ion sources and acceleration mechanisms inferred from local distribution functions, Geophys. Res. Lett., 24, 955.

Borovsky, J. E., M. F. Thomsen, and D. J. McComas (1997), The superdense plasma sheet: Plasmaspheric origin, solar wind origin, or ionospheric origin?, J. Geophys. Res., 102, 22,089.

Burton, R. K., R. L. McPherron, and C. T. Russell (1975), An empirical relationship between interplanetary conditions and Dst, J. Geophys. Res., $80,4204$.

Candidi, M., S. Orsini, and V. Formisano (1982), The properties of ionospheric $\mathrm{O}^{+}$ions as observed in the magnetotail boundary layer and northern plasma lobe, J. Geophys. Res., 87, 9097.

Chappell, C. R., T. E. Moore, and J. H. Waite Jr. (1987), The ionosphere as a fully adequate source of plasma for the Earth's ionosphere, J. Geophys. Res., 92, 5896.

Chappell, B. L. Giles, T. E. Moore, D. C. Delcourt, P. D. Craven, and M. O. Chandler (2000), The adequacy of the ionospheric source in supplying magnetospheric plasma, J. Atmos. Sol. Terr. Phys., 62, 421.

Cladis, J. B., and H. L. Collin (1997), Observations of long-term fieldaligned flow of $\mathrm{O}^{+}$ions near the equator during summer and winter, J. Geophys. Res., 102, 22,077.

Collin, H. L., J. M. Quinn, and J. B. Cladis (1993), An empirical static model of low-energy ring current ions, Geophys. Res. Lett., 20, 141

Cully, C. M., E. F. Donovan, A. W. Yau, and G. G. Arkos (2003a), Akebono/Suprathermal Mass Spectrometer observations of low-energy ion outflow: Dependence on magnetic activity and solar wind conditions, J. Geophys. Res., 108(A2), 1093, doi:10.1029/2001JA009200.

Cully, C. M., E. F. Donovan, A. W. Yau, and H. J. Opgenoorth (2003b), Supply of thermal ionospheric ions to the central plasma sheet, J. Geophys. Res., 108(A2), 1092, doi:10.1029/2002JA009457.

Daglis, I. A., and W. I. Axford (1996), Fast ionospheric response to enhance activity in geospace: Ion feeding of the inner magnetotail, J. Geophys. Res., 101, 5047.

Daglis, I. A., E. T. Sarris, and B. Wilken (1993), AMPTE/CCE observations of the ion population at geosynchronous altitudes, Ann. Geophys., 11,685 .

Delcourt, D. C., B. L. Giles, C. R. Chappell, and T. E. Moore (1988a), Low-energy bouncing ions in the magnetosphere: a three-dimensional numerical study of Dynamics Explorer 1 data, J. Geophys. Res., 93, 1859.
Delcourt, D. C., J. L. Horwitz, and K. R. Swinney (1988b), Influence of the interplanetary magnetic field orientation on polar cap ion trajectories: Energy gain and drift effects, J. Geophys. Res., 93, 7565.

Delcourt, D. C., C. R. Chappell, T. E. Moore, and J. H. Waite Jr. (1989), A three-dimensional numerical model of ionospheric plasma in the magnetosphere, J. Geophys. Res., 94, 11,893.

Delcourt, D. C., J. A. Sauvaud, and T. E. Moore (1990), Cleft contribution to ring current formation, J. Geophys. Res., 95, 20,937.

Delcourt, D. C., T. E. Moore, and C. R. Chappell (1994), Contribution of low-energy ionospheric protons to the plasma sheet, J. Geophys. Res., 99, 5681.

Dessler, A. J., and F. C. Michel (1966), Plasma in the geomagnetic tail, J. Geophys. Res., 71, 1421.

Eastman, T. E., L. A. Frank, W. K. Peterson, and W. Lennartsson (1984), The plasma sheet boundary layer, J. Geophys. Res., 89, 1553.

Elliott, H., R. Comfort, P. Craven, M. Chandler, and T. Moore (2001), Solar wind influence on the oxygen content of ion outflow in the high-altitude polar cap during solar minimum conditions, J. Geophys. Res., 106, 6067.

Elphic, R. C., L. A. Weiss, M. F. Thomsen, D. J. McComas, and M. B. Moldwin (1996), Evolution of plasmaspheric ions at geosynchronous orbit during times of high geomagnetic activity, Geophys. Res. Lett., 23, 2189.

Fennell, J. F., D. R. Croley, and S. M. Kaye (1981), Low energy ion pitch angle distribution in the outer magnetosphere: ion zipper distributions, J. Geophys. Res., 86, 3375.

Freeman, J. W., Jr., H. K. Hills, T. W. Hill, P. H. Reiff, and D. A. Hardy (1977), Heavy ion circulation in the Earth's magnetosphere, Geophys. Res. Lett., 4, 195.

Fu, S. Y., Q. G. Zong, T. A. Fritz, Z. Y. Pu, and B. Wilken (2002), Composition signatures in ion injections and its dependence on geomagnetic conditions, J. Geophys. Res., 107(A10), 1299, doi:10.1029/ 2001JA002006.

Fuselier, S. A., D. M. Klumpar, and E. G. Shelley (1991), Ion reflection and transmission during reconnection at the Earth's subsolar magnetopause, Geophys. Res. Lett., 18, 139.

Gosling, J. T., M. F. Thomsen, S. J. Bame, R. C. Elphic, and C. T. Russell (1990), Cold ion beams in the low latitude boundary layer during accelerated flow events, Geophys. Res. Lett., 17, 2245.

Hamilton, D. C., G. Gloeckler, F. M. Ipavich, W. Studemann, B. Wilken, and G. Kremser (1988), Ring current development during the great magnetic storm of February 1986, J. Geophys. Res., 93, 14,343.

Hirahara, M., T. Mukai, T. Terasawa, S. Machida, Y. Saito, T. Yamamoto, and S. Kokubun (1996), Cold dense ion flows with multiple components observed in the distant tail lobe by Geotail, J. Geophys. Res., 101, 7769.

Horwitz, J. L. (1982), The ionosphere as a source for magnetospheric ions, Rev. Geophys., 20, 929.

Kan, J. R., and L. C. Lee (1979), Energy coupling function and solar windmagnetosphere dynamo, Geophys. Res. Lett., 6, 577.

Kaye, S. M., E. G. Shelley, R. D. Sharp, and R. G. Johnson (1981), Ion composition of zipper events, J. Geophys. Res., 86, 3383.

Kozyra, J. U., E. G. Shelley, R. H. Comfort, L. H. Brace, T. E. Cravens, and A. F. Nagy (1987), The role of ring current $\mathrm{O}^{+}$in the formation of stable auroral red arcs, J. Geophys. Res., 92, 7487.

Kozyra, J. U., A. F. Nagy, and D. W. Slater (1997), High-altitude energy source(s) for stable auroral red arcs, Rev. Geophys., 35, 155.

Lennartsson, W., and E. G. Shelley (1986), Survey of $0.1-$ to $16-\mathrm{keV} / \mathrm{e}$ plasma sheet ion composition, J. Geophys. Res., 91, 3061.

Lennartsson, W., R. D. Sharp, E. G. Shelley, R. G. Johnson, and H. Balsiger (1981), Ion composition and energy distribution during 10 magnetic storms, J. Geophys. Res., 86, 4628.

Mitchell, D. G., P. C:son Brandt, E. C. Roelof, D. C. Hamilton, K. C. Retterer, and S. Mende (2003), Global imaging of $\mathrm{O}^{+}$from IMAGE/ HENA, Space Sci. Rev., 109, 63.

Moore, T. E., and D. C. Delcourt (1995), The geopause, Rev. Geophys., 33, 175 .

Moore, T. E., et al. (1995), The thermal ion dynamics experiment and plasma source instrument, Space Sci. Rev., 71, 409.

Moore, T. E., et al. (1997), High altitude observations of the polar wind, Science, 277, 349

Moore, T. E., W. K. Peterson, C. T. Russell, M. O. Chandler, M. R. Collier, H. L. Collin, P. D. Craven, R. Fitzenreiter, B. L. Giles, and C. J. Pollock (1999), Ionospheric mass ejection in response to a CME, Geophys. Res. Lett., 26, 2339.

Moore, T. E., B. L. Giles, D. C. Delcourt, and M.-C. Fok (2000), The plasma sheet source groove, J. Atmos. Sol. Terr. Phys., 62, 505.

Moore, T. E., et al. (2005), Plasma sheet and ring current formation from solar and polar wind sources, J. Geophys. Res., 110, A02210, doi:10.1029/2004JA010563.

Mukai, T., M. Hirahara, S. Machida, Y. Saito, T. Terasawa, and A. Nishida (1994), Geotail observation of cold ion streams in the medium distance 
magnetotail lobe in the course of a substorm, Geophys. Res. Lett., 21, 1023

Nosé, M., S. Ohtani, K. Takahashi, A. T. Y. Lui, R. W. McEntire, D. J. Williams, S. P. Christon, and K. Yumoto (2001), Ion composition of the near-Earth plasma sheet in storm and quiet intervals: Geotail/EPIC measurements, J. Geophys. Res., 106, 8391.

Nosé, M., R. W. McEntire, and S. P. Christon (2003), Change of the plasma sheet ion composition during magnetic storm development observed by the Geotail spacecraft, J. Geophys. Res., 108(A5), 1201, doi:10.1029/ 2002JA009660.

Orsini, S., M. Candidi, V. Formisano, H. Balsiger, A. Ghielmetti, and K. W. Ogilvie (1984), The structure of the plasma sheet-lobe boundary in the Earth's magnetotail, J. Geophys. Res., 89, 1573.

Orsini, S., M. Candidi, M. Stokholm, and H. Balsiger (1990), Injection of ionospheric ions into the plasma sheet, J. Geophys. Res., 95, 7915

Parks, G., L. J. Chen, M. McCarthy, D. Larson, R. P. Lin, T. Phan, H. Reme, and T. Sanderson (1998), New observations of ion beams in the plasma sheet boundary layer, Geophys. Res. Lett., 25, 3285.

Peroomian, V. (2003), The influence of the interplanetary magnetic field on the entry of solar wind ions into the magnetosphere, Geophys. Res. Lett., 30(7), 1407, doi:10.1029/2002GL016627.

Peroomian, V., and M. Ashour-Abdalla (1996), Population of the near-Earth magnetotail from the auroral zone, J. Geophys. Res., 101, 15,387.

Peterson, W. K., R. D. Sharp, E. G. Shelley, and R. G. Johnson (1981), Energetic ion composition of the plasma sheet, J. Geophys. Res., 86 , 761.

Pulkkinen, T., N. Ganushkina, D. Baker, N. Turner, J. Fennell, J. Roeder, T. Fritz, M. Grande, B. Kellett, and G. Kettmann (2001), Ring current ion composition during solar minimum and rising solar activity: Polar/ CAMMICE/MICS results, J. Geophys. Res., 106, 19,131.

Seki, K., M. Hirahara, T. Terasawa, I. Shinohara, T. Mukai, Y. Saito, S. Machida, T. Yamamoto, and S. Kokubun (1996), Coexistence of Earth-origin $\mathrm{O}^{+}$and solar wind-origin $\mathrm{H}^{+} / \mathrm{He}^{++}$in the distant magnetotail, Geophys. Res. Lett., 23, 985.

Seki, K., M. Hirahara, T. Terasawa, I. Shinohara, T. Mukai, Y. Saito, S. Machida, T. Yamamoto, and S. Kokubun (1998), Statistical properties and possible supply mechanisms of tailward cold $\mathrm{O}^{+}$beams in the lobe/mantle regions, J. Geophys. Res., 103, 4477.

Seki, K., R. Elphic, M. Thomsen, J. Bonnell, E. Lund, M. Hirahara, T. Terasawa, and T. Mukai (2000), Cold flowing $\mathrm{O}^{+}$beams in the lobe/mantle at Geotail: Does FAST observe the source?, J. Geophys Res., 105, 15,931.

Seki, K., et al. (2003), Cold ions in the hot plasma sheet of Earth's magnetotail, Nature, 422, 589

Sharp, R. D., D. L. Carr, W. K. Peterson, and E. G. Shelley (1981), Ion streams in the magnetotail, J. Geophys. Res., 86, 4639.

Sharp, R. D., W. Lennartsson, W. K. Peterson, and E. G. Shelley (1982), The origins of the plasma in the distant plasma sheet, J. Geophys. Res., $87,10,420$.

Sheldon, R. B., and H. E. Spence (1997), Alfvén boundaries: Noses and zippers, Adv. Space Res., 20(3), 445.
Shelley, E. G., R. G. Johnson, and R. D. Sharp (1972), Satellite observations of energetic heavy ions during a geomagnetic storm, J. Geophys. Res., 77, 6104.

Shelley, E. C., W. K. Peterson, A. G. Ghielmetti, and J. Geiss (1982), The polar ionosphere as a source of energetic magnetospheric plasma, Geophys. Res. Lett., 9, 12,103.

Strangeway, R. J., C. T. Russell, C. W. Carlson, J. P. McFadden, R. E Ergun, M. Temerin, D. M. Klumpar, W. K. Peterson, and T. E. Moore (2000), Cusp field-aligned currents and ion outflows, J. Geophys. Res., $105,21,129$

Stubbs, T. J., M. Lockwood, P. Cargill, M. Grande, B. Kellett, and C. Perry (2004), A comparison between ion characteristics observed by the POLAR and DMSP spacecraft in the high-latitude magnetosphere, Ann. Geophys., 22, 1033

Su, Y.-J., J. L. Horwitz, T. E. Moore, M. O. Chandler, P. D. Craven, B. L. Giles, M. Hirahara, and C. J. Pollock (1998), Polar wind survey with TIDE/PSI suite aboard Polar, J. Geophys. Res., 103, 29,305.

Su, Y.-J., J. E. Borovsky, M. F. Thomsen, R. C. Elphic, and D. J. McComas (2000), Plasmaspheric material at the reconnecting magnetopause, J. Geophys. Res., 105, 7591

Su, Y.-J., J. E. Borovsky, M. F. Thomsen, N. Dubouloz, M. O. Chandler, T. E. Moore, and M. Bouhram (2001), Plasmaspheric material on high-latitude open field lines, J. Geophys. Res., 106, 6085.

Thomsen, M. F., J. E. Borovsky, R. M. Skoug, and C. W. Smith (2003), The delivery of cold, dense plasma sheet material into the near-earth region, J. Geophys. Res., 108(A4), 1151, doi:10.1029/2002JA009544.

Williams, D. J. (1981), Energetic ion beams at the edge of the plasma sheet: ISEE 1 observations plus a simple explanatory model, J. Geophys. Res., $86,5507$.

Winglee, R. M. (2000), Mapping of ionospheric outflows into the magnetosphere for varying IMF conditions, J. Atmos. Sol. Terr. Phys., 62, 527.

Winglee, R. M. (2003), Circulation of ionospheric and solar wind particle populations during extended southward interplanetary magnetic field, J. Geophys. Res., 108(A10), 1385, doi:10.1029/2002JA009819.

Wodnicka, E. B., and M. Banaszkiewicz (1997), Outflowing ionospheric oxygen-ion motion in a reconfigurating magnetosphere, Ann. Geophys., 15,5

Young, D. T., H. Balsiger, and J. Geiss (1982), Correlations of magnetospheric ion composition with geomagnetic and solar activity, J. Geophys. Res., 87, 9077.

P. D. Craven and W. Maddox, National Space Science and Technology Center, NASA Marshall Space Flight Center, 320 Sparkman Drive, XD12, Huntsville, AL 35805, USA.

J. U. Kozyra, M. W. Liemohn, and A. F. Nagy, Atmospheric, Oceanic, and Space Science Department, University of Michigan, 2455 Hayward Street, Ann Arbor, MI 48109-2143, USA. (liemohn@umich.edu)

T. E. Moore, Laboratory for Extraterrestrial Physics, Interplanetary Physics MC 692, NASA Goddard Space Flight Center, Greenbelt, MD 20771, USA. 